Supporting Information

\title{
Computational Nanomechanics of Noncollagenous Interfibrillar Interface in Bone
}

\author{
Yang Wang ${ }^{l}$, Reza Morsali ${ }^{1}$, Zhengwei Dai ${ }^{2}$, Majid Minary-Jolandan ${ }^{l}$, and Dong Qian $^{l} *$ \\ ${ }^{I}$ Department of Mechanical Engineering, The University of Texas at Dallas, 800 W. Campbell Rd, \\ Richardson, TX 75080, USA \\ ${ }^{2}$ College of Material and Textile Engineering, Jiaxing University, Jiaxing, 314001, People's Republic of \\ China
}

*Corresponding Author, Email: dong.qian@utdallas.edu 
Mass Distribution of Each Component. Bone consists of roughly 60\% mineral (calcium and phosphate), $10-20 \%$ water and $20-30 \%$ proteins. Among the proteins, only about $10 \%$ are noncollagenous proteins including primarily OC and OPN proteins. ${ }^{1}$ Also, about $40 \%$ of mineral in bone is in the form of HAP at the interfibrillar interface..$^{2-3}$ From the periodical gap-overlap pattern of the collagen microfibrils, ${ }^{4}$ approximately $8 \%$ of the total mass of the composite model was assigned to the noncollagenous proteins. The chain numbers of the OPN and OC proteins were chosen to be 4 and 64, respectively, to maintain the mass ratio of 1:2 between OPN and OC proteins according to experimental results. ${ }^{5}$ With this treatment, the masses of OPN proteins, OC proteins, and HAP were 141505.7, 236481.2 and 4066713.3 Da, respectively. To neutralize the system, 220 $\mathrm{Ca}^{2+}$ were added to the model. As a result, OPN, OC, HAP and $\mathrm{Ca}^{2+}$ ions took up $3.2 \%, 5.3 \%$, $91.3 \%$ and $0.2 \%$ of the total weight, respectively. Other parameters of the composite interface model are provided in Table S1.

Table S1. Geometric properties of the composite model.

\begin{tabular}{c|c}
\hline Number of chains & 4 OPN $+64 \mathrm{OC}+4048$ HAP \\
\hline Number of neutralized ions & $220 \mathrm{Ca}^{2+}$ \\
\hline Number of total atoms & 228848 \\
\hline Interfacial area, $\mathrm{A}_{0}\left(\mathrm{~nm}^{2}\right)$ & 171.1 \\
\hline Hight, $\mathrm{h}(\mathrm{nm})$ & 8.2 \\
\hline Volume $\left(\mathrm{nm}^{3}\right)$ & 1403.0 \\
\hline
\end{tabular}




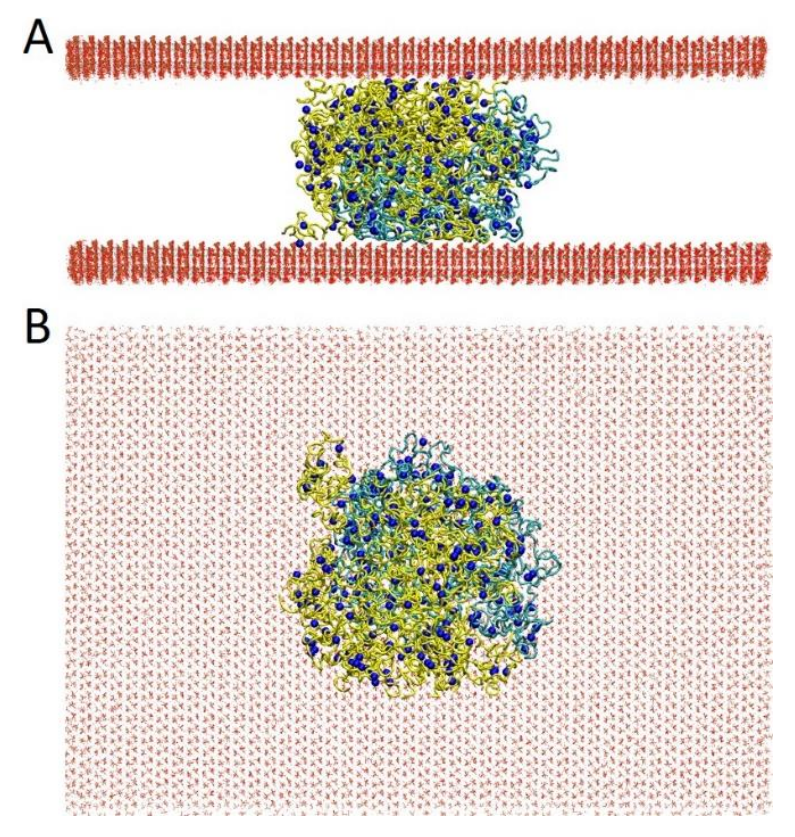

Figure S1. Initial configuration of the composite model. (A) Side view of the composite model. (B) Top view of the composite model. The cyan chains are the OPN proteins, the yellow ones are the $\mathrm{OC}$ proteins and the blue dots represent the $\mathrm{Ca}^{2+}$ ions. The initial gap between the two HAP platelets is $8.2 \mathrm{~nm}$.

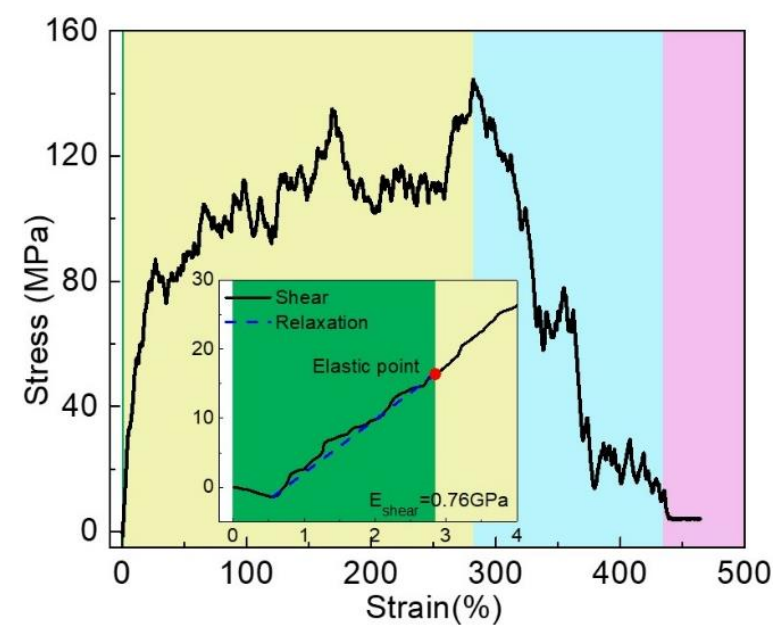

Figure S2. Stress-strain curve for the composite model under shear. The inset shows the zoom-in shear stress-strain response up to $4.0 \%$ shear strain. The elastic limit (shown as the red dot) corresponds to the maximum shear strain value at which the applied shear deformation is fully recoverable. The corresponding shear strain is found to be $2.9 \%$. Shear modulus of $762.6 \mathrm{Ma}$ is obtained from the slope by linearly fitting of the stress-strain curve up to the elastic limit. 
Binding Sites at the Atomic Scale. The [001] HAP surface has abundant $\mathrm{Ca}^{2+}$ and $\mathrm{PO}^{4-}$ ions. The OC and OPN proteins are rich in aspartic acid (ASP) and glutamic acid (GLU) residues. These residues are negatively charged and tend to adhere to the HAP surface through positively charged $\mathrm{Ca}^{2+}$ ions as shown in Figure S3A, B and D. Besides ASP and GLU, arginine acid (ARG) also serves as another major binding site to HAP (Figure S3C and E). More specifically, hydrogen atoms from $\mathrm{ARG}$ in $\mathrm{OC}$ proteins interact with oxygen atoms from $\mathrm{PO}^{4-}$ in $\mathrm{HAP}$, creating hydrogen bonds as shown in Figure S3C and E. Apart from the binding sites between HAP and noncollagenous proteins, the $220 \mathrm{Ca}^{2+}$ ions added to the system for neutralization also form the electrostatic bonds to link the noncollagenous proteins as shown in Figure S4.

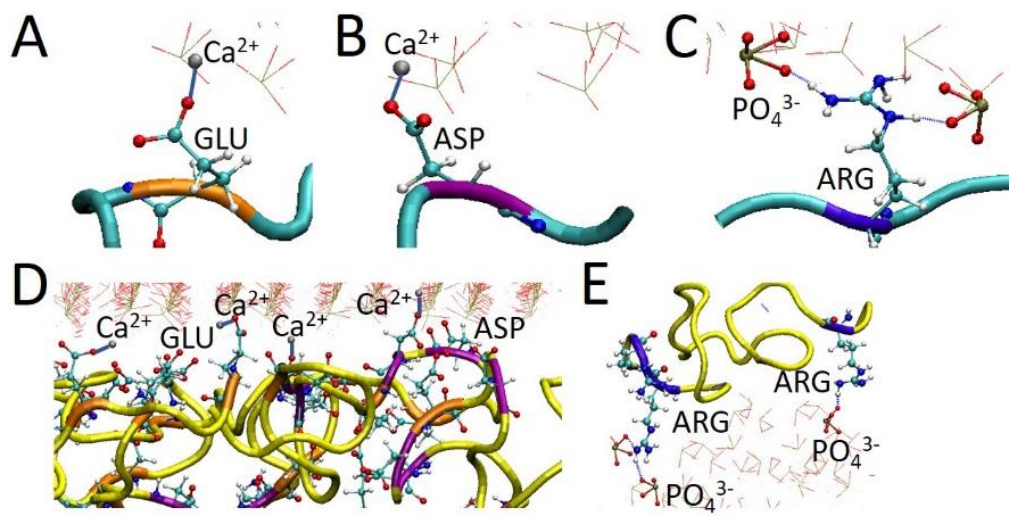

Figure S3. Bindings between (A) GLU residue in OPN proteins and $\mathrm{Ca}^{2+}$ ions in HAP, (B) ASP residue in OPN and $\mathrm{Ca}^{2+}$ ions in HAP, (C) ARG residue in OPN and oxygen atoms of $\mathrm{PO}_{4}{ }^{3-}$ in HAP, (D) GLU and ASP in OC proteins and $\mathrm{Ca}^{2+}$ ions in HAP, (E) ARG residue in OC proteins and oxygen atoms of $\mathrm{PO}_{4}{ }^{3-}$ in $\mathrm{HAP}$. 


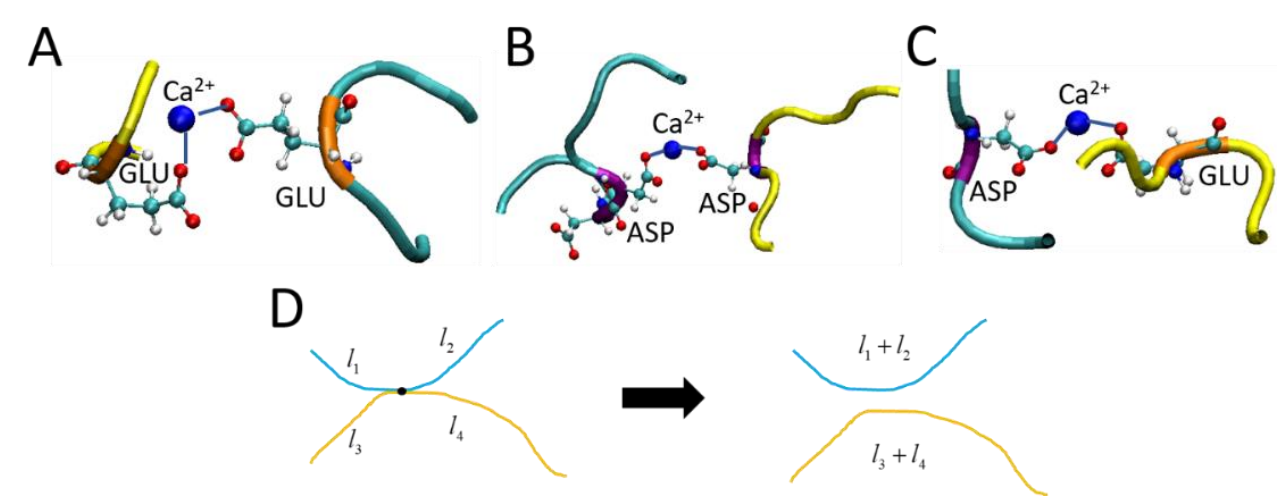

Figure S4. Binding mechanisms between the OC and OPN proteins. (A-C) Negatively charged GLU/ASP from OPN proteins is binded to GLU/ASP from OC proteins through $\mathrm{Ca}^{2+}$ ions. (D) A schematic plot showing merge of the protein segments due to the breakage of the interchain interactions which leads the protein chains to a low-entropy conformational state.
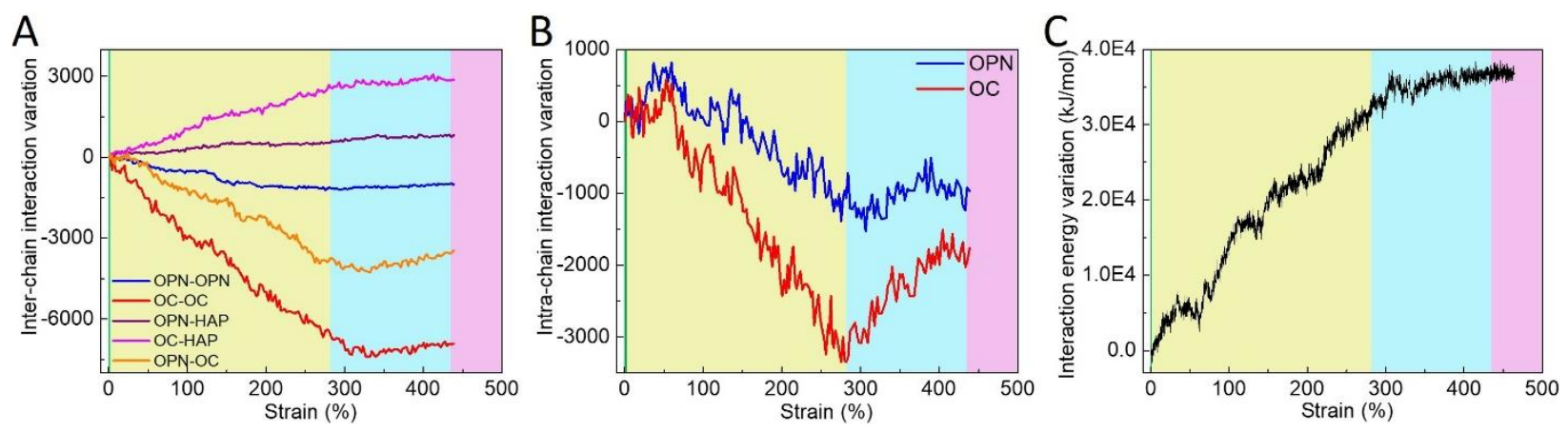

Figure S5. (A) Changes in the interchain interactions among different components as functions of applied shear strain. (B) Changes in the intrachain interactions within OPN and OC proteins as functions of shear strain. (C) Sum of the nonbonded interaction energy variation of OPN-OPN and OC-OC.

Gyration Radius Analysis at the Chain Scale. Gyration radius $R_{g}$ is defined as the average of the root mean square distance of each atom from the mass center of the chain. For comparison purposes, all calculated $R_{g}$ values were normalized to their initial values. The OPN3 and OC19 molecules were chosen for illustrating the deformation mechanisms. 
Characterization of the Sacrificial Bond, Hidden Length and Free Length. Sacrificial bonds were identified by detecting a series of nonsequential backbone atom pairs that form nonbonded intrachain interactions. The piecewise protein chains separated by the sacrificial bonds provided the hidden lengths and the free lengths depending on whether the corresponding segment is inside or outside of the pair of sacrificial bonds.

Bonded and Nonbonded Interaction Energy. The bonded interaction energy was evaluated by summing up bond stretch, bond angle and bond dihedral energy as shown in Figure S6. Nonbonded interaction energy consists of the electrostatic energy and van der Waals energy.

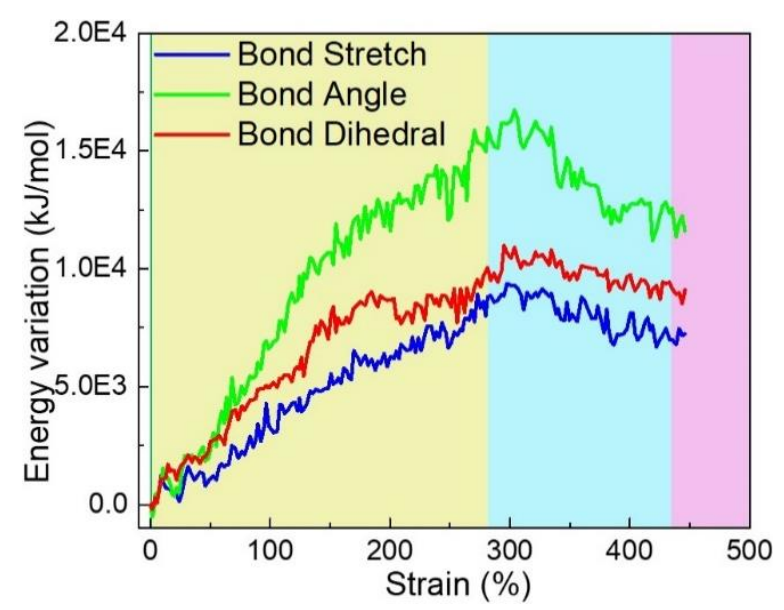

Figure S6. Changes in the stretch, bending and twist terms of the bonded energy as functions of shear strain. 


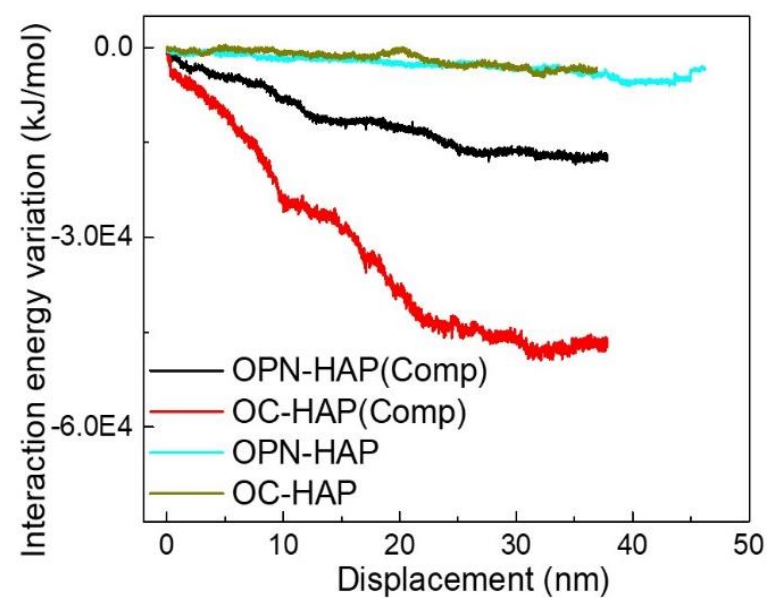

Figure S7. Changes in the interaction energy between the OC proteins and HAP, and between the OPN proteins and HAP as part of the composite interface, and the corresponding ones in the singecomponent systems. 


\section{SI Reference}

(1) Barthelat, F.; Yin, Z.; Buehler, M. J. Structure and mechanics of interfaces in biological materials. Nat. Rev. Mater. 2016, 1 (4), 16007.

(2) Lees, S.; Prostak, K.; Ingle, V.; Kjoller, K. The loci of mineral in turkey leg tendon as seen by atomic force microscope and electron microscopy. Calcif. Tissue Int. 1994, 55 (3), 180-189.

(3) Nair, A. K.; Gautieri, A.; Chang, S.-W.; Buehler, M. J. Molecular mechanics of mineralized collagen fibrils in bone. Nat. Commun. 2013, 4, 1724.

(4) Hall, J. E. Guyton and Hall textbook of medical physiology e-Book, Elsevier Health Sciences: 2015.

(5) Poundarik, A. A.; Diab, T.; Sroga, G. E.; Ural, A.; Boskey, A. L.; Gundberg, C. M.; Vashishth, D. Dilatational band formation in bone. Proc. Natl. Acad. Sci. 2012, 109 (47), 19178-19183. 